\title{
Developing the evidence base for accreditation of healthcare organisations: a call for transparency and innovation
}

\author{
David Greenfield, Jeffrey Braithwaite
}

Why has so little evidence of the efficacy of accreditation programmes been published in the peer-reviewed literature? Since its introduction in the 1970s accreditation has spread across the world to become an established part of healthcare systems in over 70 countries and there is an associated international body. ${ }^{1}$ It is a textbook case of Everett Rogers' Diffusion of innovation, whereby an idea whose time has come is adopted first by early proponents, then is received by the majority, and then becomes normal practice. ${ }^{2}$ However, the empirical evidence base for accreditation programmes is substantially undeveloped. It is not as if the challenge to publish evidence has been missing. There have been many calls in the international literature for research into accreditation. ${ }^{3-6}$ So while it is accepted that accreditation programmes have been an important driver to improve quality and safety in healthcare organisations, ${ }^{378}$ a rigorous, transparent examination of different aspects of accreditation, and publication of the subsequent results, has not become the norm.

Nevertheless, accreditation agencies continue to revise their programmes or standards and introduce new methods. ${ }^{7-9}$ These initiatives are potentially important, and seem to reflect considerable improvements. The test for accreditation agencies is to publish in the peer-reviewed literature their evidence and rationale for change and improvement. At present this is uncommon. By way of example let us take two newer unrelated methods that have been recently implemented, or being considered by accreditation agencies: unannounced surveys and tracer methodology.

Institute of Health Innovation, Centre for Clinical Governance Research, Faculty of Medicine, University of New South Wales, Kensington, New South Wales, Australia

Correspondence to: David Greenfield, Institute of Health Innovation, Centre for Clinical Governance Research, Faculty of Medicine, University of New South Wales, 10 Arthur St, Kensington NSW 2052, Australia; d.greenfield@unsw.edu.au
Unannounced surveys, sometimes called short notice surveys, are external visits where the organisation under review has no prior notice that this is to take place. They have been introduced into the practice of accreditation in the $\mathrm{USA}^{10}$ and are being considered for use in Australia. ${ }^{11}$ A range of claims have been made about their benefits. These include that they allow an organisation to focus on patient care rather than preparing for the next scheduled survey. In addition, it is thought that external organisations and the public view short notice surveys more credibly than scheduled surveys. While this may be true, a review of the published peer-reviewed literature could find no systematic or rigorous empirical evidence to support these claims. ${ }^{12}$

Tracer methodologies have a history as long as accreditation programmes, having been introduced into healthcare in the early 1970 s. ${ }^{13}$ More recently a distinction was made between "patient system tracers" and "patient care tracers". ${ }^{14}$ The former focus on processes, functions and systems which are understood to affect patient care. The latter target patients, examining their trajectory through the health system and the care standards with which they intersect. When used within an accreditation survey, surveyors have the task of considering the findings simultaneously to assess the quality and safety of the health organisation. ${ }^{15}{ }^{16}$ Some empirical studies have been conducted on tracer methodologies; at last count, we reviewed eight research papers. The methodology is deemed in this research to be a useful method for assessing the quality of care. However, none of these studies examined the use of tracer methodologies within an accreditation survey or programme. ${ }^{12}$

Let us be clear: it is good that programme revisions and new initiatives are being introduced by accreditation agencies. We argue that the continuous improvement ideal should be applied to accreditation, and the capacity of accred- itation agencies to embrace new ideas seems to us to be increasing. But we remain troubled. Our concern is that new initiatives are being adopted without a higher level of transparency. Where is the evidence base that we demand of clinicians? And should not new methods or initiatives be introduced as pilot projects that are publicly evaluated, then moved into full-scale implementation with rigorous evaluation, and the findings published in the peer-reviewed literature?

Things may be changing. Research into accreditation is underway. ${ }^{17}$ Accreditation agencies in the UK, Europe, Australia and Canada are engaged in research programmes. ${ }^{1}$ What is not quite so clear is how transparent agencies will be with their results. The challenge for accreditation agencies is to publish their research protocols and then their findings, both positive and negative, in the peer-reviewed literature. When they do publish their results the agencies will be explicitly displaying leadership, transparency and evidence of improvement, the very qualities and conduct they require of the health organisations they accredit. In an evidence-based world, should we demand anything less?

Competing interests: None.

Qual Saf Health Care 2009;18:162-163.

doi:10.1136/qshc.2009.032359

\section{REFERENCES}

1. Greenfield D, Braithwaite J. Health sector accreditation research: a systematic review. Int J Qual Health Care 2008;20:172-83.

2. Rogers E. Diffusion of innovations, 5th edn. New York: Free Press, 2003.

3. Baker S, Dunn D. Accreditation: the hallmark of educational quality. Radiol Technol 2006;78:123-30.

4. Fernandopulle R, Ferris T, Epstein A, et al. A research agenda for bridging the "quality chasm". Health Aff 2003;22:178-90.

5. Ovretveit J, Gustafson D. Improving the quality of health care: using research to inform quality programmes. BMJ 2003;326:759-61.

6. Shaw C. External assessment of health care. BMJ 2001;322:851-4.

7. Australian Council on Healthcare Standards. The Australian Council on Healthcare Standards Annual Report 2007-2008. Sydney: Australian Council on Healthcare Standards, 2008.

8. Health Information and Quality Authority. Standards health \& social services. http://www.hiqa ie/functions_sh_ss.asp (accessed 14 Jan 2009).

9. Joint Commission. Accreditation process overview. http://www.jointcommission.org/AboutUs/Fact Sheets/ overview_qa.htm (accessed 14 Jan 2009).

10. Joint Commission. Unannounced surveysrationale and benefits. http://www.jointcommission. org/AccreditationPrograms/Unannounced_Surveys. htm (accessed 14 Jan 2009).

11. Australian Commission on Quality and Safety in Healthcare. An alternative model for safety and quality accreditation (draft). Sydney: Australian Commission on Quality and Safety in Healthcare, 2007.

12. Greenfield D, Travaglia J, Braithwaite J, et al. Unannounced surveys and tracer methodology: 
literature review. Sydney: Centre for Clinical

Governance Research, 2007.

13. Kessner D, Kalk C. A strategy for evaluating health services. Washington DC: Institute of Medicine,

National Academy of Sciences, 1973.

14. Joint Commission on Accreditation of

Healthcare Organizations. Joint Commission renames tracer activities. Joint Commission

Perspectives 2005:25:9-10.

15. DeLorenzo M. Shared visions-new pathways: what to expect at your next JCAHO survey. Nurs Manage 2005:36:26-30.

16. Friedman M. Tracer methodology and the new Joint Commission home care and hospice survey process: part 1. Home Healthc Nurse

2004;22:710-14.

17. Braithwaite J, Westbrook J, Pawsey M, et al. A prospective, multi-method, multi-disciplinary, multilevel, collaborative, social-organisational design for researching health sector accreditation. BMC Health Serv Res 2006;6:113. 